\title{
Linear Pursuit Differential Game under Phase Constraint on the State of Evader
}

\author{
Askar Rakhmanov, ${ }^{1}$ Gafurjan Ibragimov, ${ }^{2}$ and Massimiliano Ferrara ${ }^{3,4}$ \\ ${ }^{1}$ Department of Informatics, Tashkent University of Information Technologies, TUIT, Amir Temur Str. 108, \\ 100202 Tashkent, Uzbekistan \\ ${ }^{2}$ Department of Mathematics \& Institute for Mathematical Research, Universiti Putra Malaysia, Serdang, Malaysia \\ ${ }^{3}$ Department of Law and Economics, University Mediterranea of Reggio Calabria, Reggio Calabria, Italy \\ ${ }^{4}$ ICRIOS, Bocconi University, Milan, Italy
}

Correspondence should be addressed to Massimiliano Ferrara; massimiliano.ferrara@unirc.it

Received 22 June 2016; Accepted 14 September 2016

Academic Editor: Rigoberto Medina

Copyright (C) 2016 Askar Rakhmanov et al. This is an open access article distributed under the Creative Commons Attribution License, which permits unrestricted use, distribution, and reproduction in any medium, provided the original work is properly cited.

We consider a linear pursuit differential game of one pursuer and one evader. Controls of the pursuer and evader are subjected to integral and geometric constraints, respectively. In addition, phase constraint is imposed on the state of evader, whereas pursuer moves throughout the space. We say that pursuit is completed, if inclusion $y\left(t_{1}\right)-x\left(t_{1}\right) \in M$ is satisfied at some $t_{1}>0$, where $x(t)$ and $y(t)$ are states of pursuer and evader, respectively, and $M$ is terminal set. Conditions of completion of pursuit in the game from all initial points of players are obtained. Strategy of the pursuer is constructed so that the phase vector of the pursuer first is brought to a given set, and then pursuit is completed.

\section{Introduction}

Pursuit and evasion differential game problems under different type of constraints on controls of players were studied by many researchers, for example, [1-24]. In [11, 16], main approaches of solution of pursuit and evasion differential games were given and fundamental results were obtained. In $[2-5,7,10,13,15,17-19,22,24]$, various pursuit and evasion differential game problems were investigated under phase constraints. Sufficient conditions of completion of pursuit were obtained in $[4,5,19]$, when controls of both players are subjected to geometric constraints [19] or integral constraints [4], and phase constraint is being imposed on the state of the evader. The objectives of the researches $[8,9,12]$ are to find value of the game and construct optimal strategies of players. The paper [5] is devoted to discrete pursuit games with phase constraints.

The present paper is continuation of $[4,5,19]$ and deals with the linear pursuit differential games where pursuit control is subjected to integral constraints and evasion control is subjected to geometric constraint, with phase constraint being imposed on the state of the evader. For such constraints on controls of players, to obtain solution of pursuit problem for all initial states of phase space is complicated because of boundedness of pursuit resources.

In the present paper, the following technique is proposed to control the phase vector of the pursuer: first-phase vector of the pursuer is brought to a given bounded, convex set, regardless of the behavior of evading player and then, using the evasion control and initial positions of the players, the pursuit control that ensures the completion of pursuit is constructed.

New sufficient conditions of completion of pursuit from all initial positions for a finite time have been obtained and results have been illustrated on a model example. This paper is closely related to $[4,5,19,20,23]$.

\section{Statement of Problem}

Consider a linear differential game of one pursuer and one evader described by

$$
\begin{aligned}
& \dot{x}=A x+u, \\
& \dot{y}=B y+v,
\end{aligned}
$$


where $x, y \in R^{n}$ are state vectors of pursuer and evader, respectively, $A, B$ are $n \times n$ matrices, and $u, v \in R^{n}$ are control parameters of pursuer and evader, respectively.

The terminal set has the form $M=M_{0}+M_{1}$, where $M_{0}$ is a linear subspace of $R^{n}$ and $M_{1}$ is a subset of $L$, which is orthogonal complement of $M_{0}$ to $R^{n}$. It is assumed that relative interior of $M_{0}$ with respect to $L$ is not empty. Pursuer moves throughout the space, but evader is subjected to state constraint and it moves only within given bounded convex set $D$.

Definition 1. Measurable functions $u=u(t)$ and $v=v(t)$, $t \geq 0$, that satisfy constraints

$$
\begin{aligned}
\int_{0}^{\infty}|u(t)|^{2} d t & \leq \rho^{2}, \\
v & \in Q \subset R^{n},
\end{aligned}
$$

are called admissible controls of pursuer and evader, respectively, where $\rho>0$ is a given number and $Q$ is a compact subset of $R^{n}$.

Definition 2. One says that pursuit can be completed from initial points $x_{0}, y_{0} \in R^{n}, y_{0} \in D, y_{0}-x_{0} \notin M$, for time $t_{1}$; if, for any admissible control of evader $v=v(t), 0 \leq t \leq t_{1}$, one can construct an admissible control of pursuer $u=u(t)$, $0 \leq t \leq t_{1}$, such that, for solutions $x(t), y(t), 0 \leq t \leq t_{1}$, of initial value problems,

$$
\begin{aligned}
& \dot{x}=A x+u(t), \quad x(0)=x_{0}, \\
& \dot{y}=B y+v(t), \quad y(0)=y_{0} .
\end{aligned}
$$

Inclusion $y\left(t_{1}\right)-x\left(t_{1}\right) \in M$ is satisfied and $y(t) \in D$ for all $0 \leq t \leq t_{1}$. It is assumed that, to construct pursuit control $u(t)$, it is allowed to use values $v(t), x(t), y(t)$ at any time $t \geq 0$.

As it is known, if $u=u(t)$ and $v=v(t), t \geq 0$, are any admissible controls of players, then, for the initial value problems (3), we have

$$
\begin{aligned}
& x(t)=\Psi(t) x_{0}+\int_{0}^{t} \Psi(t-\tau) u(\tau) d \tau \\
& y(t)=\Phi(t) y_{0}+\int_{0}^{t} \Phi(t-\tau) v(\tau) d \tau,
\end{aligned}
$$

where $\Psi(t)=\exp (A t), \Phi(t)=\exp (B t), t \geq 0$.

Problem 3. Find conditions of completion of pursuit in game (1)-(2) from all initial points of players.

\section{Main Result}

Let $\pi$ denote the operator of orthogonal projection of $R^{n}$ onto $L$. Since int $M_{1} \neq \varnothing$, set $D$ is convex and bounded, $Q$ is compact set, and then there exist numbers $\ell>0, R>0, \sigma>0$, and vector $m \in M_{1}$, such that $D \subset R S \subset R^{n}, \ell S_{1} \subset-m+M_{1}$, $Q \subset \sigma S$, where $S$ and $S_{1}$ are closed unit balls of spaces $R^{n}$ and $L$ and centered at the origins of these spaces, respectively. It is clear that, in the case of $R \leq \ell$, solution of pursuit problem is straightforward, and, therefore, from now on, we assume that $R \gg \ell$.

Assumption 4. There exist numbers $d>0, \ell_{1}, \ell_{2} \geq 0, \ell_{1}+\ell_{2}=$ $\ell, \alpha, 1<\alpha \leq R /\left(R-\ell_{2}\right)$ and a linear measurable mapping $F(t, \tau): R^{n} \rightarrow R^{n}$ in $\tau, 0 \leq \tau \leq t$, such that

$$
\begin{aligned}
& \text { (i) }\|F(t, \tau)\| \leq d, 0 \leq \tau \leq t, \\
& \text { (ii) }(1 / \alpha) \int_{0}^{t}[\pi \Phi(t-\tau)-\pi \Psi(t-\tau) F(t, \tau)] Q d \tau \subset \ell_{1} S_{1} .
\end{aligned}
$$

Assumption 5. There exist continuous function $T:(0, \rho) \rightarrow$ $[0, \infty)$ and number $\rho_{1}, 0<\rho_{1}<\rho$, such that

(i) $\left(\rho-\rho_{1}\right)^{2} / T\left(\rho_{1}\right)>d^{2} \sigma^{2} / \alpha^{2}$,

(ii) for each pair of points $\left(x_{*}, y\right), y-x_{*} \notin M, y \in D$, $x_{*} \in D$, there is time $t=t\left(x_{*}, y\right) \leq T\left(\rho_{1}\right)$ for

$$
\begin{gathered}
-m+\frac{1}{\alpha} \pi \Phi(t) y-\pi \Psi(t) x_{*} \\
\in \int_{0}^{t} \pi \Psi(t-\tau)\left(\frac{\rho_{1}}{\sqrt{t}} S\right) d \tau,
\end{gathered}
$$

where $x_{*} \in D$ and this point can be, in particular, the center of the ball of minimal radius containing $D$.

It follows from Assumption 5(i) that there is number $\varepsilon$, $0<\varepsilon<\rho-\rho_{1}$, such that

$$
\frac{\left(\rho-\rho_{1}-\varepsilon\right)^{2}}{T\left(\rho_{1}\right)}>\frac{d^{2} \sigma^{2}}{\alpha^{2}} .
$$

Assumption 6. For any $x \in R^{n}$, there is finite number $r=$ $r(x)>0$ such that

$$
-x_{*}+\Psi(r) x \in \int_{0}^{r} \Psi(r-\tau)\left(\sqrt{\frac{2 \rho \varepsilon-\varepsilon^{2}}{r}}\right) S d \tau .
$$

Theorem 7. Let Assumptions 4-6 hold true. Then, pursuit can be completed in game (1)-(2) from all points $\left(x_{0}, y_{0}\right), y_{0}-x_{0} \notin$ $M, y_{0} \in D$, for a finite time.

Proof. Let $\left(x_{0}, y_{0}\right), y_{0}-x_{0} \notin M, y_{0} \in D$ be any point. Consider two cases: (i) $x_{0} \in D$ and (ii) $x_{0} \notin D$. Let $x_{0} \in D$. According to Assumption 5(ii), for pair $\left(x_{0}, y_{0}\right), y_{0}-x_{0} \notin M, x_{0} \in D$, specify time $t_{1}=t_{1}\left(x_{0}, y_{0}\right) \leq T\left(\rho_{1}\right)$, satisfying

$$
\begin{gathered}
-m+\frac{1}{\alpha} \pi \Phi\left(t_{1}\right) y_{0}-\pi \Psi\left(t_{1}\right) x_{0} \\
\in \int_{0}^{t_{1}} \pi \Psi\left(t_{1}-\tau\right)\left(\frac{\rho_{1}}{\sqrt{t_{1}}} S\right) d \tau .
\end{gathered}
$$

It follows from (8) that there exists measurable selection $w(t)$, $0 \leq t \leq t_{1}$, of multivalued mapping $\pi \Psi\left(t_{1}-\tau\right)\left(\left(\rho_{1} / \sqrt{t_{1}}\right) S\right)$, $0 \leq \tau \leq t_{1}$, such that

$$
-m+\frac{1}{\alpha} \pi \Phi\left(t_{1}\right) y_{0}-\pi \Psi\left(t_{1}\right) x_{0}=\int_{0}^{t_{1}} w(t) d t .
$$


Now, we consider

$$
w(t)=\pi \Psi\left(t_{1}-t\right) \omega(t), \quad 0 \leq t \leq t_{1},
$$

with respect to unknown vector-function $\omega(t) \in\left(\rho_{1} / \sqrt{t_{1}}\right) S$, $0 \leq t \leq t_{1}$. Then, we can apply Filippov's Lemma [6] on existence of measurable solution of (10).

Therefore,

$$
\begin{gathered}
-m+\frac{1}{\alpha} \pi \Phi\left(t_{1}\right) y_{0}-\pi \Psi\left(t_{1}\right) x_{0} \\
=\int_{0}^{t_{1}} \pi \Psi\left(t_{1}-t\right) \omega(t) d t
\end{gathered}
$$

with respect to unknown vector-function $\omega(t) \in\left(\rho_{1} / \sqrt{t_{1}}\right) S$, $0 \leq t \leq t_{1}$, has measurable solution $\omega=\omega_{1}^{0}(t), 0 \leq t \leq t_{1}$.

Next, assuming that $v=v(t), 0 \leq t \leq t_{1}$, is any admissible control of the evader, we construct pursuit control as follows:

$$
u(t)=u_{1}(t)=\frac{1}{\alpha} F\left(t_{1}, t\right) v(t)+\omega_{1}^{0}(t), \quad 0 \leq t \leq t_{1} .
$$

Then, using (4) yields

$$
\begin{aligned}
& \frac{1}{\alpha} \pi y\left(t_{1}\right)-\pi x\left(t_{1}\right)=\frac{1}{\alpha} \pi \Phi\left(t_{1}\right) y_{0}-\pi \Psi\left(t_{1}\right) x_{0}+\frac{1}{\alpha} \\
& \quad \cdot \int_{0}^{t_{1}}\left[\pi \Phi\left(t_{1}-\tau\right)-\pi \Psi\left(t_{1}-\tau\right) F\left(t_{1}, \tau\right)\right] v(\tau) d \tau \\
& \quad-\int_{0}^{t_{1}} \pi \Psi\left(t_{1}-\tau\right) \omega_{1}^{0}(\tau) d \tau=\frac{1}{\alpha} \\
& \quad \cdot \int_{0}^{t_{1}}\left[\pi \Phi\left(t_{1}-\tau\right)-\pi \Psi\left(t_{1}-\tau\right) F\left(t_{1}, \tau\right)\right] v(\tau) d \tau \\
& \quad+\frac{1}{\alpha} \pi \Phi\left(t_{1}\right) y_{0}-\pi \Psi\left(t_{1}\right) x_{0} \\
& \quad-\int_{0}^{t_{1}} \pi \Psi\left(t_{1}-\tau\right) \omega_{1}^{0}(\tau) d \tau .
\end{aligned}
$$

Next, adding vector $-m$ to both sides of (13) and then using Assumption 4(ii) and (12) yield

$$
\begin{aligned}
& -m+\frac{1}{\alpha} \pi y\left(t_{1}\right)-\pi x\left(t_{1}\right)=-m+\frac{1}{\alpha} \pi \Phi\left(t_{1}\right) y_{0} \\
& -\pi \Psi\left(t_{1}\right) x_{0}-\int_{0}^{t_{1}} \pi \Psi\left(t_{1}-t\right) \omega_{1}^{0}(t) d t+\frac{1}{\alpha} \\
& . \int_{0}^{t_{1}}\left[\pi \Phi\left(t_{1}-\tau\right)-\pi \Psi\left(t_{1}-\tau\right) F\left(t_{1}, \tau\right)\right] v(\tau) d \tau \\
& =\frac{1}{\alpha} \int_{0}^{t_{1}}\left[\pi \Phi\left(t_{1}-t\right)-\pi \Psi\left(t_{1}-t\right) F\left(t_{1}, t\right)\right] v(t) d t \\
& \in \frac{1}{\alpha} \int_{0}^{t_{1}}\left[\pi \Phi\left(t_{1}-\tau\right)-\pi \Psi\left(t_{1}-\tau\right) F\left(t_{1}, \tau\right)\right] Q d \tau \\
& \subset \ell_{1} S_{1} .
\end{aligned}
$$

Therefore,

$$
\left|-m+\frac{1}{\alpha} \pi y\left(t_{1}\right)-\pi x\left(t_{1}\right)\right| \leq \ell_{1}
$$

Now, we use inclusion $y(T) \in D \subset R S$, inequality (15), and definition of numbers $\alpha, \ell_{1}, \ell_{2}$ to obtain

$$
\begin{aligned}
& \left|-m+\pi y\left(t_{1}\right)-\pi x\left(t_{1}\right)\right| \\
& \quad=\left|\pi y\left(t_{1}\right)-\frac{1}{\alpha} \pi y\left(t_{1}\right)-m+\frac{1}{\alpha} \pi y\left(t_{1}\right)-\pi x\left(t_{1}\right)\right| \\
& \quad \leq \frac{\alpha-1}{\alpha}\left|\pi y\left(t_{1}\right)\right|+\left|-m+\frac{1}{\alpha} \pi y\left(t_{1}\right)-\pi x\left(t_{1}\right)\right| \\
& \quad \leq \frac{\alpha-1}{\alpha} R+\ell_{1} \leq \ell_{2}+\ell_{1}=\ell .
\end{aligned}
$$

Therefore,

$$
\pi y\left(t_{1}\right)-\pi x\left(t_{1}\right) \in m+\ell S_{1} \subset M_{1},
$$

which implies that

$$
y\left(t_{1}\right)-x\left(t_{1}\right) \in M
$$

Thus, we have proven that, in case (i), game (1)-(2) is completed at time $t_{1}$.

Let us turn to case (ii). By Assumption 6, for point $x_{0} \in$ $R^{n}$, the following inclusion is satisfied at some time $r_{1}=$ $r_{1}\left(x_{0}\right)>0$ :

$$
-x_{*}+\Psi\left(r_{1}\right) x_{0} \in \int_{0}^{r_{1}} \Psi\left(r_{1}-\tau\right)\left(\sqrt{\frac{2 \rho \varepsilon-\varepsilon^{2}}{r_{1}}}\right) S d \tau .
$$

Consider

$$
-x_{*}+\Psi\left(r_{1}\right) x_{0}=\int_{0}^{r_{1}} \Psi\left(r_{1}-\tau\right) u(\tau) d \tau
$$

with respect to unknown vector-function $u(t) \quad \epsilon$ $\left(\sqrt{\left(2 \rho \varepsilon-\varepsilon^{2}\right) / r_{1}}\right) S, 0 \leq t \leq r_{1}$. Using Filippov's lemma [6], we obtain from inclusion (19) existence of measurable solution of (20). Denote it by $u_{0}(t), 0 \leq t \leq r_{1}$. Let $t=r_{1}$, $u(t)=-u_{0}(t), 0 \leq t \leq r_{1}$, in (3) and use (20) with $u(t)=u_{0}(t)$, $0 \leq t \leq r_{1}$, to obtain

$$
\begin{aligned}
x\left(r_{1}\right)= & x_{*}-x_{*}+\Psi\left(r_{1}\right) x_{0} \\
& -\int_{0}^{r_{1}} \Psi\left(r_{1}-\tau\right) u_{0}(\tau) d \tau=x_{*} \in D .
\end{aligned}
$$

Now, take $t=r_{1}$ as the initial time and let $x_{0}=x\left(r_{1}\right)=x_{*}$, $y_{0}=y\left(r_{1}\right)$. Assume that $y_{0}-x_{0} \notin M$, since game (1)-(2) is completed at $t=r_{1}$. Then, conditions of case (i) are satisfied and the rest of the proof runs as before. It should be noted that, in case (ii), pursuit is completed at the time $r_{1}+t_{1}$.

Next, we show admissibility of control (12). Indeed, using the Minkowski inequality gives

$$
\begin{aligned}
& \int_{0}^{t_{1}}\left|u_{1}(t)\right|^{2} d t=\int_{0}^{t_{1}}\left|\frac{1}{\alpha} F\left(t_{1}, t\right) v(t)+\omega_{1}^{0}(t)\right|^{2} d t \\
& \leq\left[\left(\int_{0}^{t_{1}}\left|\frac{1}{\alpha} F\left(t_{1}, t\right) v(t)\right|^{2} d t\right)^{1 / 2}\right. \\
& \left.\quad+\left(\int_{0}^{t_{1}}\left|\omega_{1}^{0}(t)\right|^{2} d t\right)^{1 / 2}\right]^{2} .
\end{aligned}
$$


Then, by Assumptions 4(i) and 5, according to $\omega_{1}^{0}(t) \in$ $\left(\rho_{1} / \sqrt{t_{1}}\right) S, Q \subset \sigma S$, we have, in case (i), that

$$
\begin{aligned}
\int_{0}^{t_{1}}\left|u_{1}(t)\right|^{2} d t & \leq\left[\frac{d \sigma}{\alpha} \sqrt{t_{1}}+\left(\frac{\rho_{1}}{t_{1}}\right) t_{1}\right]^{2} \\
& \leq\left[\frac{d \sigma}{\alpha} \sqrt{T\left(\rho_{1}\right)}+\rho_{1}\right]^{2} \\
& <\left(\rho-\rho_{1}+\rho_{1}\right)^{2}=\rho^{2},
\end{aligned}
$$

and, in case (ii),

$$
\begin{aligned}
\int_{0}^{t_{1}}\left|u_{1}(t)\right|^{2} d t & \leq\left[\frac{d \sigma}{\alpha} \sqrt{t_{1}}+\left(\frac{\rho_{1}}{t_{1}}\right) t_{1}\right]^{2} \\
& \leq\left[\frac{d \sigma}{\alpha} \sqrt{T\left(\rho_{1}\right)}+\rho_{1}\right]^{2} \\
& <\left(\rho-\varepsilon-\rho_{1}+\rho_{1}\right)^{2}=(\rho-\varepsilon)^{2} .
\end{aligned}
$$

Since, by construction $\left|u_{0}(t)\right| \leq \sqrt{\varepsilon(2 \rho-\varepsilon) / r_{1}}, 0 \leq t \leq r_{1}$,

$$
\begin{aligned}
\int_{0}^{r_{1}}\left|u_{0}(t)\right|^{2} d t & \leq \varepsilon(2 \rho-\varepsilon), \\
\int_{0}^{r_{1}+t_{1}}|u(t)|^{2} d t & =\int_{0}^{r_{1}}\left|u_{0}(t)\right|^{2} d t+\int_{r_{1}}^{r_{1}+t_{1}}\left|u_{1}(t)\right|^{2} d t \\
& =\varepsilon(2 \rho-\varepsilon)+(\rho-\varepsilon)^{2}=\rho^{2},
\end{aligned}
$$

which implies admissibility of pursuit control in case (ii). Theorem 7 has been proven.

Assumption 8. There exist numbers $d_{1}>0, \alpha_{1}, 1<\alpha_{1} \leq$ $R /(R-\ell)$ and a linear measurable mapping $F_{1}(t, \tau): R^{n} \rightarrow R^{n}$ in $\tau, 0 \leq \tau \leq t$, such that

(i) $\pi \Psi(t-\tau) F_{1}(t, \tau)=\pi \Phi(t-\tau), 0 \leq \tau \leq t$,

(ii) Assumptions 4(i) and 5 are satisfied at $\alpha=\alpha_{1}, d=d_{1}$.

Theorem 9. Let Assumptions 6 and 8 hold true. Then, pursuit can be completed in game (1)-(2) from any point $\left(x_{0}, y_{0}\right), y_{0}-$ $x_{0} \notin M, y_{0} \in D$ for a finite time.

Proof. Let $\left(x_{0}, y_{0}\right), y_{0}-x_{0} \notin M, y_{0} \in D$ be an arbitrary point. As in the proof of Theorem 7, there are two cases: (i) $x_{0} \in D$, and (ii) $x_{0} \notin D$. Let $x_{0} \in D$. Using Assumption 5(ii), we define time $t_{2}=t_{2}\left(x_{0}, y_{0}\right) \leq T\left(\rho_{1}\right)$ for point $\left(x_{0}, y_{0}\right), y_{0}-x_{0} \notin M$, that satisfies

$$
\begin{array}{r}
-m+\frac{1}{\alpha_{1}} \pi \Phi\left(t_{2}\right) y_{0}-\pi \Psi\left(t_{2}\right) x_{0} \\
\epsilon \int_{0}^{t_{2}} \pi \Psi\left(t_{2}-\tau\right)\left(\frac{\rho_{1}}{\sqrt{t_{2}}} S\right) d \tau .
\end{array}
$$

Consider

$$
\begin{gathered}
-m+\frac{1}{\alpha_{1}} \pi \Phi\left(t_{2}\right) y_{0}-\pi \Psi\left(t_{2}\right) x_{0} \\
=\int_{0}^{t_{2}} \pi \Psi\left(t_{2}-t\right) \omega(t) d t,
\end{gathered}
$$

with respect to unknown vector-function $\omega(t) \in\left(\rho_{1} / \sqrt{t_{2}}\right) S$, $0 \leq t \leq t_{2}$. Using Filippov's lemma [6], we obtain from (26) that there exists a measurable solution of (27). Denote it by $\omega_{2}^{0}(t), 0 \leq t \leq t_{2}$.

Let $v=v(t), 0 \leq t \leq t_{2}$, be any admissible control of the evader. For each $t \in\left[0, t_{2}\right]$, we construct pursuit control as follows:

$$
u(t)=u_{2}(t)=\frac{1}{\alpha_{1}} F\left(t_{2}, t\right) v(t)+\omega_{2}^{0}(t), \quad 0 \leq t \leq t_{2} .
$$

Then, using Assumption 8(i) and the fact that $\omega_{2}^{0}(t), 0 \leq t \leq$ $t_{2}$, is a solution of (27), we obtain, for solution (4) of initial value problem (3) at $t=t_{2}$,

$$
\begin{aligned}
& -m+\pi y\left(t_{2}\right)-\pi x\left(t_{2}\right)=-m+\pi \Phi\left(t_{2}\right) y_{0}-\pi \Psi\left(t_{2}\right) \\
& \cdot x_{0}+\int_{0}^{t_{2}}\left(\pi \Phi\left(t_{2}-t\right)-\frac{1}{\alpha_{1}} \pi \Psi\left(t_{2}-t\right) F_{1}\left(t_{2}, t\right)\right) \\
& \cdot v(t) d t-\int_{0}^{t_{2}} \pi \Psi\left(t_{2}-t\right) \omega_{2}^{0}(t) d t=-m+\frac{1}{\alpha_{1}} \\
& \cdot \pi \Phi\left(t_{2}\right) y_{0}-\pi \Psi\left(t_{2}\right) x_{0}+\left(1-\frac{1}{\alpha_{1}}\right)\left[\pi \Phi\left(t_{2}\right) y_{0}\right. \\
& \left.+\int_{0}^{t_{2}} \pi \Phi\left(t_{2}-t\right) v(t) d t\right]-\int_{0}^{t_{2}} \Psi\left(t_{2}-t\right) \omega_{2}^{0}(t) d t \\
& =\left(1-\frac{1}{\alpha_{1}}\right) \pi y\left(t_{2}\right) .
\end{aligned}
$$

Since, by Assumption 8, $1<\alpha_{1} \leq R /(R-\ell)$ and $y\left(t_{2}\right) \in D \subset$ $R S$, (29) implies that

$$
\begin{aligned}
& -m+\pi y\left(t_{2}\right)-\pi x\left(t_{2}\right)=\left(1-\frac{1}{\alpha_{1}}\right) \pi y\left(t_{2}\right) \\
& \in\left(1-\frac{1}{\alpha_{1}}\right) R S \subset \ell S .
\end{aligned}
$$

Hence,

$$
\pi y\left(t_{2}\right)-\pi x\left(t_{2}\right) \in M_{1} .
$$

Let us turn to case (ii). By Assumption 6, for any point $x_{0} \in$ $R^{n}$, there is time $r_{2}=r_{2}\left(x_{0}\right)>0$ such that inclusion (19) is satisfied at $r_{1}=r_{2}$. The same reasoning as that in the proof of Theorem 7 applies to conclude that $x\left(r_{2}\right)=x_{*} \in D$. If now $t=r_{2}$ is taken as the initial time for the game and set $x_{0}=x\left(r_{2}\right)=x_{*}, y_{0}=y\left(r_{2}\right)$, then we arrive at case (i), and so there exists $t_{2}>0$ such that $\pi y\left(t_{2}\right)-\pi x\left(t_{2}\right) \in M_{1}$. Note that, in case (ii), in order to complete the game, the pursuer has to spend time $r_{2}+t_{2}$.

Thus, it has been proven that control (28) guarantees completion of pursuit in game (1)-(2) at $r_{2}+t_{2}$, which proves the theorem.

Admissibility of pursuit control (28) can be obtained in much the same way as in the proof of Theorem 7.

From Theorems 7 and 9, one can easily obtain the following statements, respectively. 
Corollary 10. Let, for point $\left(x_{0}, y_{0}\right), y_{0}-x_{0} \notin M, x_{0}, y_{0} \in D$, there exists time $t_{3}=t_{3}\left(x_{0}, y_{0}\right)>0$ such that Assumptions 4 and 5 are satisfied at $t=t_{3}, x_{*}=x_{0}, y=y_{0}$. Then, pursuit can be completed from point $\left(x_{0}, y_{0}\right)$ at time $t_{3}$.

Corollary 11. Let, for point $\left(x_{0}, y_{0}\right), y_{0}-x_{0} \notin M, x_{0}, y_{0} \in D$, there exists time $t_{4}=t_{4}\left(x_{0}, y_{0}\right)>0$ such that Assumption 8 is satisfied at $t=t_{4}, x_{*}=x_{0}, y=y_{0}$. Then, pursuit can be completed from point $\left(x_{0}, y_{0}\right)$ at time $t_{4}$.

Thus, Corollaries 10 and 11 enable us to draw conclusion that if the pursuer is in set $D$ at the initial time, then completion of pursuit is guaranteed without Assumption 6.

Example 12. Consider a simple motion differential game:

$$
\begin{aligned}
& \dot{x}=u, \\
& \dot{y}=v,
\end{aligned}
$$

where $x, y, u, v \in R^{n}, n \geq 1$, pursuit control function is a measurable function subject to integral constraint:

$$
\int_{0}^{\infty}|u(t)|^{2} d t \leq \rho^{2}
$$

and evasion control function is a measurable function subject to geometric constraint:

$$
|v| \leq \sigma
$$

A phase constraint is imposed to the state of the evader $y$

$$
y \in R S \subset R^{n} .
$$

Differential game (32)-(34) is considered completed if $y-x \in$ $\ell S, \ell>0$; that is, $M=\ell S \subset R^{n}$.

It is not difficult to verify that if

$$
\rho>2 \sqrt{\sigma(R-\ell)},
$$

then, for game (32) with different type constraints (33)(35), all the hypotheses of Assumptions 4-8 are satisfied. Therefore, Theorems 7 and 9 can be applied and they give the same result, namely, if inequality (36) is satisfied, then pursuit is completed in game (32) with constraints (33)-(35) from all initial points $y_{0}-x_{0} \notin M, y_{0} \in R S$, for a finite time. Note that if there is not a state constraint for the evader, then pursuit can be completed in game (32)-(34) only from some points of the phase space.

\section{Conclusion}

We have proposed a technique to solve pursuit problem which consists of two steps: first-step state of the pursuer is brought to a given bounded convex set, regardless of the behavior of evading player and then, in the second step, pursuit is completed.

New sufficient conditions of completion of pursuit from all initial positions for a finite time have been obtained. Moreover, results have been illustrated on a model example.
Note that Assumptions 4, 5, and 8 depend on linear measurable function $F(t, \tau): R^{n} \rightarrow R^{n}$ of $\tau, 0 \leq \tau \leq t$, to a considerable extent. Therefore, future investigations can be carried out towards the choice of this function to weaken Assumptions 4, 5, and 8.

\section{Competing Interests}

The authors declare that there are no competing interests regarding the publication of this paper.

\section{References}

[1] A. A. Azamov, A. Sh. Kuchkarov, and B. B. Samatov, "The relation between problems of pursuit, controllability and stability in the large in linear systems with different types of constraints," Prikladnaya Matematika i Mekhanika, vol. 71, no. 2, pp. 259263, 2007.

[2] A. A. Azamov, "On the problem of kind for the simple motion game with restriction," Serdika: Bulgarsko Matematichesko Spisanie, vol. 12, pp. 38-43, 1986.

[3] A. A. Azamov, "On the evasion problem for a given curve," Applied Mathematics and Mechanics, no. 4, pp. 694-697, 1982.

[4] A. Raxmanov and G. Ibragimov, "Linear discrete pursuit game with phase constraints," Scientific World Journal, vol. 2014, Article ID 435103, 5 pages, 2014.

[5] A. Raxmonov and G. I. Ibragimov, "An approach for solving discrete game problems with total constraints on controls," Abstract and Applied Analysis, vol. 2014, Article ID 674651, 6 pages, 2014.

[6] A. F. Filippov, "On some problems of theory of optimal regulation," Vestnik Moskovskogo Universiteta, Seriya Matematiki, Mekhaniki, no. 2, pp. 25-32, 1959.

[7] G. I. Ibragimov, "A game problem on a closed convex set," Siberian Advances in Mathematics, vol. 12, no. 3, pp. 16-31, 2002.

[8] G. I. Ibragimov, "On the optimal pursuit game of several pursuers and one evader," Prikladnaya Matematika i Mekhanika, vol. 62, no. 2, pp. 199-205, 1998.

[9] G. I. Ibragimov, A. A. Azamov, and M. Khakestari, "Solution of a linear pursuit-evasion game with integral constraints," ANZIAM Journal, vol. 52, pp. E59-E75, 2010.

[10] R. P. Ivanov, "Simple pursuit-evasion on a compact convex set," Doklady Akademii Nauk SSSR, vol. 254, no. 6, pp. 1318-1321, 1980.

[11] N. N. Krasovskii, Control of Dynamic Systems, Nauka, Moscow, Russia, 1985.

[12] A. S. Kuchkarov, G. I. Ibragimov, and M. Khakestari, “On a linear differential game of optimal approach of many pursuers with one evader," Journal of Dynamical and Control Systems, vol. 19, no. 1, pp. 1-15, 2013.

[13] A. S. Kuchkarov, "On a differential game with integral and phase constraints," Automation and Remote Control, vol. 74, no. 1, pp. $12-25,2013$.

[14] M. S. Nikol'skii, "A direct method of solution of linear differential pursuit-evasion games," Mathematical Notes of the Academy of Sciences of the USSR, vol. 33, no. 6, pp. 455-458, 1983.

[15] A. H. Nishanov and A. T. Rakhmanov, "On a method of prosecution under state constraints on the state of the evader," European Applied Sciences, no. 6, pp. 48-51, 2013.

[16] L. S. Pontryagin, Selected Works, Nauka, Moscow, Russia, 1988. 
[17] B. N. Pshenichnii, A. A. Chikrii, and J. S. Rappoport, "Pursuit by several controlled objects in the presence of phase constraints," Doklady Akademii Nauk SSSR, vol. 259, no. 4, pp. 138-141, 1981.

[18] L. A. Petrosyan, "On a family of differential games of survival in space," Doklady Sciences of the USSR, vol. 161, no. 1, pp. 52-54, 1965.

[19] A. T. Rakhmanov, "Linear pursuit differential game under phase constraint on the state of evader," in Proceedings of the 7th International Conference on Priority Directions in Science and Technology in the XXI-th Century, vol. 2, pp. 123-127, Tashkent, Uzbekistan, 2014.

[20] A. T. Rahmanov, "On a pursuit method in linear differential games with integral constraints on controls," Differential Equations, vol. 25, no. 5, pp. 785-790, 1989.

[21] N. Yu. Satimov, Methods of Solution of Pursuit Problems in the Theory of Differential Games, NUUz Press, Tashkent, Uzbekistan, 2003.

[22] B. T. Samatov, "The differential game with 'a survival zone' with different classes of admissible control functions," in Game Theory and Applications, vol. 13, pp. 143-150, Nova Science, Hauppauge, NY, USA, 2008.

[23] B. T. Samatov, "The pursuit-evasion problem under integralgeometric constraints on pursuer controls," Automation and Remote Control, vol. 74, no. 7, pp. 1072-1081, 2013.

[24] L. P. Yugay, "On differential games with constraints on phase state and controls of players," Doklady Academy of Sciences of USSSR, no. 4, pp. 5-6, 1977. 


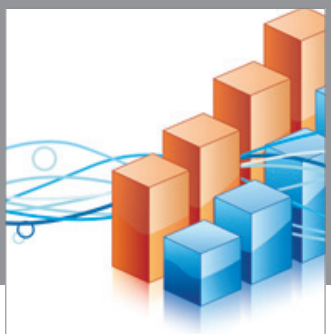

Advances in

Operations Research

vatem alat4

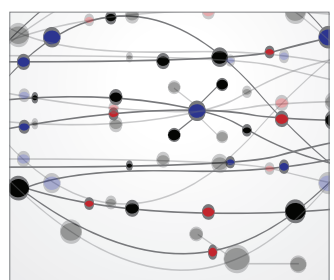

\section{The Scientific} World Journal
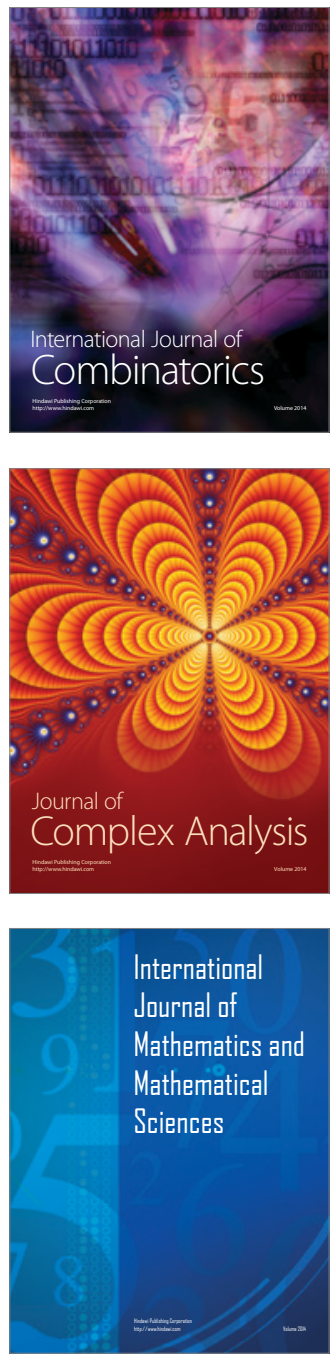
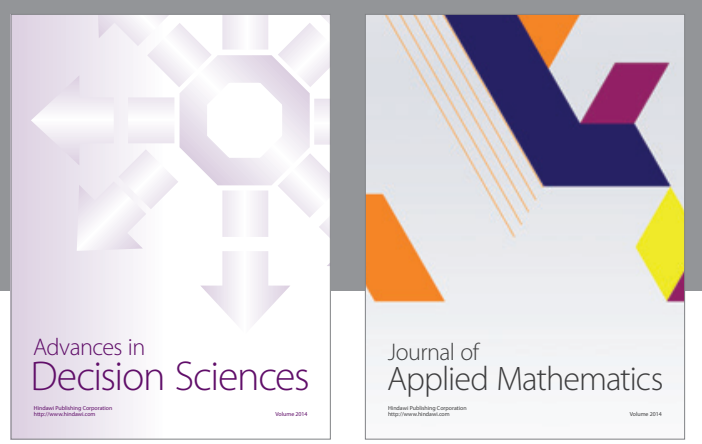

Algebra

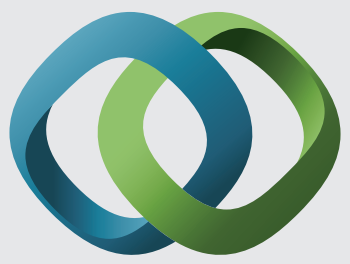

\section{Hindawi}

Submit your manuscripts at

http://www.hindawi.com
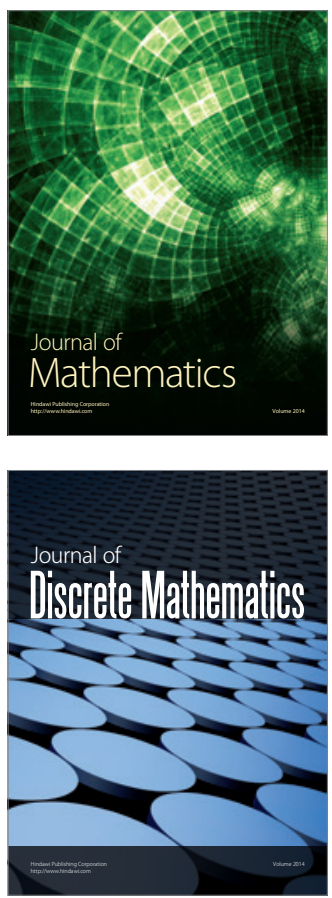

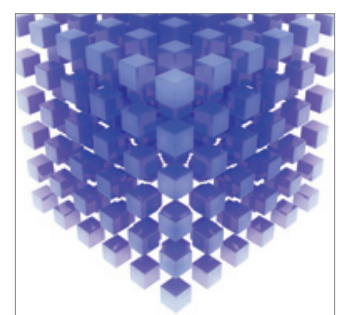

Mathematical Problems in Engineering
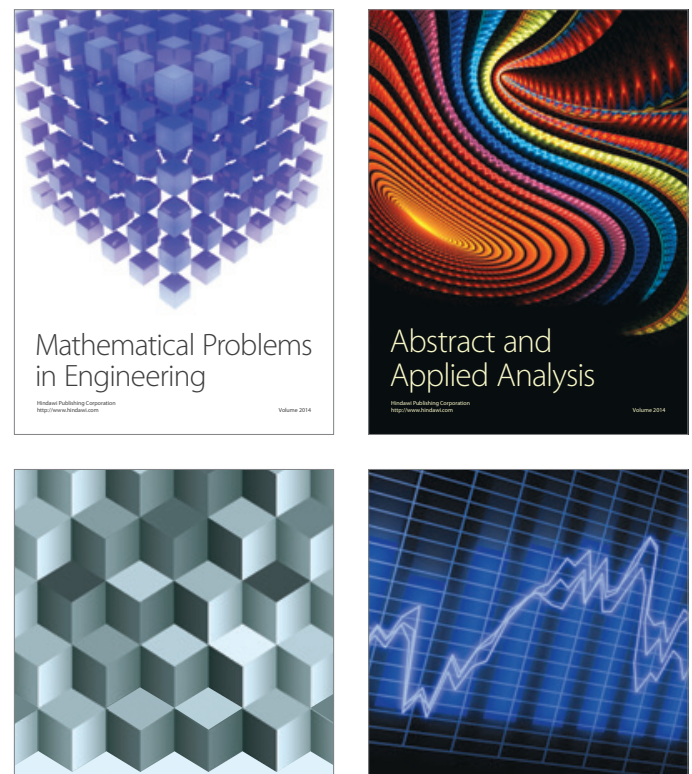

Journal of

Function Spaces

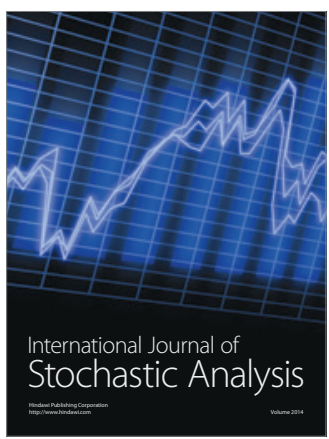

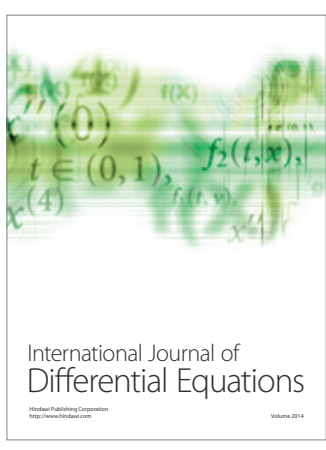
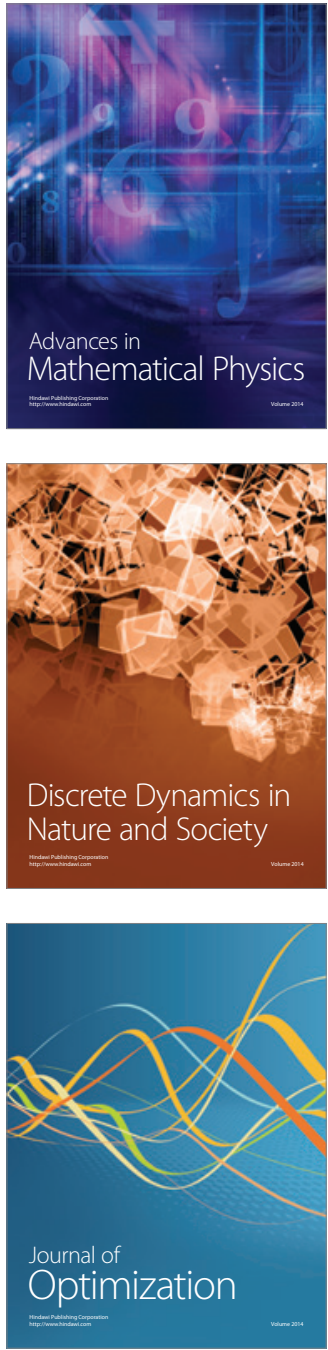